\title{
Similarity to the self influences cortical recruitment during impression formation
}

\author{
Eric D. Leshikar ${ }^{1,2}$ • Brittany S. Cassidy ${ }^{2,3}$ - Angela H. Gutchess ${ }^{2}$
}

Published online: 11 November 2015

(C) Psychonomic Society, Inc. 2015

\begin{abstract}
Prior work has shown that whether or not someone is similar to the self influences person memory - a type of self-reference effect for others. In this study, we were interested in understanding the neural regions supporting the generation of impressions and subsequent memory for targets who vary in similarity to the self. Participants underwent fMRI scanning while forming positive or negative impressions of face-behavior pairs. We tested participants' memory for their generated impressions and then back-sorted the impression trials (encoding) into different levels of self-similarity (high, medium, low) using a self-similarity posttest that came after recognition. Extending prior behavioral work, our data confirmed our hypothesis that memory would be highest for selfsimilar others and lowest for self-dissimilar others. Dorsal anterior cingulate cortex (dACC) activity increased with self-similarity (high $>$ medium $>$ low) to targets, regardless of later memory for them. An analysis of regions supporting impression memory revealed a double dissociation within medial temporal lobe regions: for similar others, amygdala recruitment supported memory, whereas for dissimilar others, hippocampal activation supported memory. These results
\end{abstract}

Electronic supplementary material The online version of this article (doi:10.3758/s13415-015-0390-3) contains supplementary material, which is available to authorized users.

Eric D. Leshikar

leshikar@uic.edu

1 Department of Psychology, University of Illinois at Chicago, 1007 West Harrison Street (M/C 285), Chicago, IL 60607-7137, USA

2 Department of Psychology, Brandeis University, Waltham, MA, USA

3 Department of Psychological and Brain Sciences, Indiana University Bloomington, Bloomington, IN, USA suggest that self-similarity influences evaluation and memory for targets but also affects the underlying neural resources engaged when thinking about others who vary in selfsimilarity.

Keywords Self-similarity · Impression formation · Memory · fMRI $\cdot$ Medial prefrontal cortex · Amygdala $\cdot$ Hippocampus . Self-reference effect

Forming and remembering impressions of others is an important ability because it enables the navigation of complex social environments. Because forming impressions of others is subjective, the content of one's self-schema (i.e., how a person views the self) influences interpersonal evaluation (Catrambone, Beike, \& Niedenthal, 1996). Indeed, one's self-schema influences many aspects of our perception of others (Amodio \& Frith, 2006), including how we initially evaluate (Catrambone \& Markus, 1987; Markus, Smith, \& Moreland, 1985) and subsequently remember others (Leshikar \& Gutchess, 2015; Leshikar, Park, \& Gutchess, 2014). In this investigation we are interested in how similarity to the self affects the cortical response while thinking about others who vary in degree of self-similarity (high, medium, low).

Although some fMRI studies have examined cortical regions supporting self-other similarity, the majority of these studies have defined similarity in a binary fashion (similar/ dissimilar; although, see Krienen, Tu, \& Buckner, 2010, Experiment 2), treating target individuals as either similar or dissimilar to the self (Benoit, Gilbert, Volle, \& Burgess, 2010; Jenkins, Macrae, \& Mitchell, 2008; Krienen et al., 2010; Mitchell, Macrae, \& Banaji, 2006; Moore, Merchant, Kahn, \& Pfeifer, 2014). Although this approach has been successful in understanding brain activity relevant to social processing, 
this approach is limiting because it does not account for the shades of similarity that can exist between the self and a target individual. This is surprising given that there are many perspectives in the social psychology literature suggesting that self-other similarity, or overlap, exists along a continuum (Aron, Aron, \& Smollan, 1992; Berscheid, Snyder, \& Omoto, 1989; Bogardus, 1933; Triandis \& Triandis, 1960, among others) rather than a simple dichotomy. For instance, a prominent framework suggests that interpersonal closeness should be measured in a continuous fashion (Aron et al., 1992). Given these rich frameworks, it is surprising that the majority of the fMRI investigations examining self-other similarity have not examined this in a more continuous fashion. An initial step to merge prior neuroimaging work with behavioral frameworks of self-similarity involves adding another level of self-other similarity (i.e., low, medium, and high self-similarity). Another limitation of this previous fMRI work examining self-other similarity is the operational definition of similarity along a single dimension (assertiveness, Bruch, Kaflowitz, \& Berger, 1988; extroversion, Fong \& Markus, 1982; political leaning, Krienen et al., 2010; masculinity, Markus et al., 1985). This does not capture the wider range of characteristics by which a target might be similar or dissimilar to oneself.

Abundant evidence implicates many cortical midline structures (CMS), such as medial prefrontal, anterior cingulate, and posterior cingulate cortex in both self-reflective thought (Northoff \& Bermpohl, 2004; Northoff et al., 2006) and thinking about others (D'Argembeau et al., 2007; Decety \& Sommerville, 2003; Lombardo et al., 2010). Recent work has focused on a more nuanced understanding of the neural architecture that supports thinking about self-similar others. This work has largely implicated the ventromedial prefrontal cortex (vmPFC) as crucial to thinking about self-similar others (Benoit et al., 2010; Ebner et al., 2011; Jenkins et al., 2008; Krienen et al., 2010; Mitchell et al., 2006; Moore et al., 2014). For instance, making inferences about the beliefs of targets who were either self-similar or dissimilar, as defined by liberal/conservative viewpoints, elicits differential mPFC activations: vmPFC activity supports inferences about selfsimilar others while dorsal medial prefrontal cortex (dmPFC) activity supports inferences about self-dissimilar others (Mitchell et al., 2006). This provided initial evidence suggesting functional specificity, dependent on self-similarity, of medial prefrontal cortex in relation to thinking about others. That vmPFC is especially responsive to self-similar others is intriguing because other evidence suggests that vmPFC activity also underlies thoughts focused on the self. Supporting this idea, previous work has shown vmPFC activity occurs both when thinking about the self and thinking about similar others (Jenkins et al., 2008). These findings point to a role of vmPFC while thinking about self-similar others. However, whether this region would be sensitive to different levels of self- similarity (i.e., high, medium, low) is unknown. Identifying brain regions that track, or code, for self-similarity is conceptually interesting because it would suggest a process dedicated to an online social-cognitive process by which other people are compared to the self.

Existing work identifies other medial prefrontal regions potentially responsive to different levels of self-similarity that may play a role in an online process of self-comparison to others. Strong support for a ventral to dorsal mPFC gradient exists, with ventral regions responsive to self-related thinking and dorsal regions responsive to other-related thinking (Denny, Kober, Wager, \& Ochsner, 2012; Qin \& Northoff, 2011; Wagner, Haxby, \& Heatherton, 2012; although, see D'Argembeau et al., 2012, for an alternative account). This suggests that ventral midline regions, such as $\mathrm{vmPFC}$, are potentially only responsive to highly self-similar targets but that more dorsal regions might respond to thinking about both similar and dissimilar others (e.g., gradients of self-similarity). Dorsal regions, for instance, could contribute to the evaluation of others for self-similarity as part of a role in processing salient self-related information. Indeed, a small but growing literature shows that $\mathrm{dmPFC}$ responds to degrees of selfrelevance (Moran, Macrae, Heatherton, Wyland, \& Kelley, 2006; Northoff et al., 2009; Phan et al., 2004; Schneider et al., 2008). Related work demonstrates that both dmPFC and dorsal anterior cingulate cortex (dACC) contribute to a process of making self-other comparisons (Northoff et al., 2006). This suggests that dorsal, and not necessarily ventral, midline regions might code for different levels of self-similarity, although no work to date has directly investigated this prospect. The present study was designed to address this gap in the literature.

A second aim of this study was to assess how selfsimilarity could impact the encoding of impressions into memory. Remembering impressions (e.g., remembering that you have positive feelings toward that person) is important when thinking about others, and several brain regions have been implicated in this process. One traditional way to test impression memory involves presenting participants with sets of to-be-integrated information about a target (e.g., face with behavior) and testing memory for information bound to that person (Cassidy \& Gutchess, 2012a; Gilron \& Gutchess, 2012; Mitchell, Macrae, \& Banaji, 2004), such as trait information (Todorov \& Uleman, 2003) or the general positive/ negative impression they formed of that target (Leshikar \& Gutchess, 2015; Leshikar et al., 2014). Neuroimaging findings show that $\mathrm{dmPFC}$ as well as temporoparietal junction activity supports successful impression encoding (Gilron \& Gutchess, 2012; Mitchell et al., 2004; Mitchell et al., 2006).

Medial temporal regions also could be involved in binding information to a target. Given its role in associating information together into memory (Cohen et al., 1999; Eichenbaum, 2000), the hippocampus might be expected to support 
memory for information bound to a target. However, another medial lobe structure, the amygdala, might play a crucial role in memory for these type of stimuli. For instance, an amnesiac with hippocampal damage but intact amygdalae successfully learned whether faces were paired with positive or negative behaviors, but patients with hippocampal lesions that extended to the amygdalae failed to learn these pairings (Todorov \& Olson, 2008). This suggests a critical role for amygdala engagement when binding social information to target individuals. Extending this work, the amygdala might also support memory for self-similar others. Supporting this possibility, the amygdala is responsive to self-relevant information (Rameson, Satpute, \& Lieberman, 2010; Yoshimura et al., 2009), and also plays an important role in impression formation (Schiller, Freeman, Mitchell, Uleman, \& Phelps, 2009).

In sum, we examined brain regions supporting the evaluation of, and subsequent memory for, targets varying in selfsimilarity (high, medium, low). We had two primary objectives. First, we identified regions responsive to thinking about others as self-similarity increased (or decreased). Specifically, we expected increased dorsal medial prefrontal cortex activity with more self-similarity. However, when defining similarity in a binary fashion (i.e., similar versus dissimilar) we predicted that vmPFC activity would support thinking about similar others consistent with prior work (Benoit et al., 2010; Jenkins et al., 2008; Mitchell et al., 2006; Moore et al., 2014). Second, we examined regions supporting memory for targets varying in self-similarity. We expected that dmPFC activity would support memory for targets similar to the self, consistent with prior work (Gilron \& Gutchess, 2012; Mitchell et al., 2004), and also predicted that the amygdala would support memory for self-similar others.

\section{Method}

\section{Participants}

Eighteen adults ( $M$ age: $22.4 S D: 2.6$; range 18-27; 9 females) recruited from Brandeis University and the Boston community participated. Participants were right-handed, native-English speakers with normal or corrected-to-normal vision, and none reported present or past incidence of neurological or psychiatric disorders. No participant reported psychoactive, vasoactive, or illegal drug use. Participants gave their informed, written consent in a protocol approved by the Institutional Review Boards at Brandeis University and Massachusetts General Hospital.

\section{Stimuli}

We used 216 faces (Minear \& Park, 2004) and 216 behaviortrait pairs in this experiment. The behavior-trait pairs were adapted from previously normed materials (Uleman, 1988, unpublished raw data). Each behavior-trait pair contained a behavior (e.g., "This person asked where the stars come from.") that implied a trait (e.g., curious). Each trait word was unique (i.e., did not repeat across the stimulus set) and was paired with only one behavior. Half of the behaviors were selected to elicit positive impressions and half negative, as determined by piloting the stimuli with an independent sample of participants. Across participants, behaviors were counterbalanced to appear equally often with female and male faces during study.

\section{Procedure}

Participants completed all procedures in one experimental session. There were three phases of the experiment: study (impression formation), test (recognition memory), and posttest used to sort the study phase trials into high, medium and low self-similarity. Before entering the scanner, participants practiced the study and test phases of the experiment for familiarization with task instructions. After practicing, participants formed impressions over three scanning runs. Each run contained 48 trials for a total of 144 impression formation (study) trials. For each study trial, a face and behavior were shown for $4,250 \mathrm{~ms}$, followed by a $250 \mathrm{~ms}$ fixation (See Fig. 1). Participants were told to form a positive or a negative impression for each trial based on the face and behavior. Participants were told there were no correct answers while forming impressions. It was this positive/negative endorsement that participants' were asked to remember for the subsequent impression memory test. Participants used the index and middle fingers of their right hand to indicate their impression (positive/negative) using an MRI-compatible response pad. After each study run, participants completed an unscanned recognition test. Retrieval sessions were administered while participants were in the scanner, as they were interspersed with study runs. We chose to intersperse study-test blocks because piloting suggested that participants had poorer memory if they encoded all 144 study trials before the memory test, and we wanted to ensure that we had sufficient trials to perform a subsequent memory analysis (impression memory hits versus misses).

Recognition memory was tested over three sessions. Overall, there were 216 recognition trials consisting of 144 studied and 72 unstudied (novel) trials. For each recognition trial, participants made two self-paced judgments: First, participants were shown a face and asked whether they generated a positive or negative impression for the face, or whether the face was new (i.e., not seen during study), by pressing a button under the first three fingers of their right hand. Impression accuracy was based on whether participants were able to remember the impression they generated during study (i.e., impression formation). Second, with the face still onscreen, participants were asked to determine which behavior had been 




Fig. 1 Trial schematic for the study and test phase of the experiment

studied with that face. Participants were shown one behavior directly below the face: on half the trials, the correct behavior that was paired with that face at study was shown, and on the remaining half of the trials, an incorrect behavior of the same valence that was paired with a different face during study was shown. Participants also had the option to say that the face was "new." Participants responded by pressing a button under the first three fingers of their right hand. Trials were separated by a $250 \mathrm{~ms}$ fixation. ${ }^{1}$ For trials where the face was novel (unstudied), participants were instructed to report "new" for this second recognition decision. Recognition trials were pseudorandomized so that no more than four new trials were presented consecutively. ${ }^{2}$

After study and test, participants completed an out of the scanner posttest that allowed us to back-sort trials into high, medium, and low self-similarity. For the posttest, participants were shown the trait word that was implied by each of the behaviors they saw during impression formation, as done previously (Leshikar \& Gutchess, 2015; Leshikar et al., 2014) and were instructed to make two self-paced judgments for

\footnotetext{
${ }^{1}$ Our prior behavioral work suggests that memory for the behaviors did not differ as a function of self (Leshikar \& Gutchess, 2015; Leshikar et al., 2014). The results of this second judgment will not be mentioned further, as our interest of this study was in the impression memory judgment.

${ }^{2}$ Trials were presented without jitter. Since all trial types occur randomly within a block, as they are based on subject-specific responses, this introduces sufficient stochasticity to our fMRI design (Henson, 2007) that allows us to generate stable estimates for our conditions of interest. Importantly, inclusion of null event/fixation trials to introduce jitter and measure inter-stimulus "baseline" is not necessary in fMRI designs if the contrast of interest is between task conditions that are randomly presented, as in our study (Henson, 2007; also see http://imaging.mrc-cbu.cam. ac.uk/imaging/DesignEfficiency). Thus, the current design allows us to assess event-related effects like impression memory effects (hits-misses) as others have done in several similar studies (Duarte, Henson, \& Graham, 2008; Dulas \& Duarte, 2011).
}

each word: First, participants rated the self-descriptiveness of the trait (e.g., Am I happy?) on a three-point scale ( $1=$ describes me a lot, 2 =describes me a little, 3 =does not describe me), and then they rated the importance of the trait when evaluating others (e.g., Is it important to know that a person is happy?) ( 1 = very important, 2 = somewhat important, $3=$ not at all important). Our prior behavioral work suggests that the importance rating does not affect memory performance (Leshikar \& Gutchess, 2015; Leshikar et al., 2014), and since our primary goal was to assess self-similarity, the importance rating will not be discussed further.

\section{fMRI acquisition}

We acquired structural and functional scans on a Siemens Avanto 1.5T whole body scanner (Siemens, Erlangen, Germany) at the Massachusetts General Hospital Martinos Center for Biological Imaging. High-resolution T1-weighted magnetization-prepared rapid gradient echo structural scans $(\mathrm{MP}-\mathrm{RAGE} ; \mathrm{TE}=3.39 \mathrm{~ms}, \mathrm{TR}=2,730 \mathrm{~ms}, 256 \times 256$ FOV) were acquired over 128 sagittal slices, $1.33-\mathrm{mm}$ thick. Functional scans were acquired using a $\mathrm{T} 2 *$-weighted echo planar imaging (EPI) sequence $(\mathrm{TR}=2,500 \mathrm{~ms}, \mathrm{TE}=40$ $\mathrm{ms}$, flip angle $=90^{\circ}, 3.1-\mathrm{mm}$ in-plane resolution), collected in 26 slices with a $10 \%$ interslice gap aligned to the anteriorposterior commissure line. A total of 104 volumes were collected during each study run which included four initial dummy scans.

\section{fMRI analysis}

Neuroimaging data were analyzed using Statistical Parametric Mapping (SPM8; Wellcome Trust Centre for Neuroimaging) implemented in MATLAB R2008a (The MathWorks Inc., 
Natick, MA). The first four dummy volumes of each study run were discarded to account for equilibration effects. We corrected the slice time acquisition of the remaining EPI volumes using the middle acquired slice as the reference and then spatially realigned all volumes to the first collected volume. The structural scan of each participant was coregistered to the mean EPI image that resulted from the realignment step and then segmented and normalized to the Montreal Neurological Institute $\mathrm{T} 1$ average brain template. These normalization parameters were then applied to all EPI volumes. The normalized volumes were resliced to $3 \mathrm{~mm} \times 3 \mathrm{~mm} \times 3 \mathrm{~mm}$ resolution and then spatially smoothed using an 8 -mm full-width at half-maximum Gaussian kernel.

Analyses of the fMRI data for the impression formation (study) trials were conducted in two steps. First, neural activity was modeled as a series of events (stick functions) coinciding with the onset of each trial type convolved with the canonical hemodynamic response function (i.e., Impression memory correct, Impression memory incorrect, Impression memory miss [e.g., saying an old face was "new"], for all three levels of self-similarity and both levels of valence). For each participant, the residual movement from the spatial realignment step was included as six covariates per session to model residual (linear) head movement. Parameter estimates for each voxel for all covariates were obtained by Restricted Maximum-Likelihood (ReML) estimation, using a high-pass temporal filter (cut-off 128 seconds) to remove low-frequency drift. Autocorrelations within each session were corrected by applying a first-order autoregressive (AR[1]) model. Over all voxels and volumes data were scaled to a grand mean of 100 (Friston, Ashburner, Kiebel, Nichols, \& Penny, 2007).

Parameter estimates for contrasts of interest for each participant were carried into a group (second) level analysis. Analyses of variance (ANOVA) were conducted on the impression formation data to examine self-similarity effects, as well as memory effects for similar relative to dissimilar others. We included estimates for high, medium, and low selfsimilarity trials split into impression correct (in which participants remembered the valence of the impression they formed at study) and impression incorrect trials (in which participants failed to remember the valence of the impression they formed at study). Data were entered into a 3 (Self-similarity: high, medium, low) $\times 2$ (Memory: impression correct, impression incorrect) model. Due to insufficient numbers of trials for every participant ( $<10$ trials), valence of the initial impression (positive, negative) was not included in the ANOVA. ${ }^{3}$ Given our interest in comparing across levels of self-similarity, we intentionally did not include a nonsocial "baseline" condition.

\footnotetext{
${ }^{3}$ Although we did not have sufficient trials to model valence at the group level, we show the proportion of impression trials as a function of selfsimilarity (high, medium, and low) and valence of the initial impression judgment (positive, negative) in Supplemental Table 1.
}

Nineteen covariates modeling subject effects (i.e., the mean across tasks for each participant) were also included in the model, to remove between-subject variance of no interest, including confounding effects of outlier data. Statistical Parametric Maps (SPMs) were created from the $t$ statistics for the various contrasts, using a pooled error estimate for all contrasts (Friston et al., 2002).

To correct for multiple comparisons, whole brain results were thresholded at $p<.005$, with a spatial extent of 19 contiguous voxels. We arrived at this study-specific threshold using a Monte Carlo simulation, which minimizes the probability of Type I and Type II errors in fMRI data (Slotnick, Moo, Segal, \& Hart, 2003). This threshold yields an equivalent statistical threshold of $p<.05$, corrected for multiple comparisons, and is analogous to the thresholding procedure used recently for impression formation work (MendeSiedlecki, Baron, \& Todorov, 2013). Where we had a priori prediction of brain activity, we also performed small volume corrections (SVC) when the region did not emerge in wholebrain effects. SVCs were implemented in SPM8 using anatomically defined regions of interest, corrected to $p<.05$, as has been done previously (Miller et al., 2008). Anatomical ROIs used for the small-volume correction were created in WFU Pickatlas (Maldjian, Laurienti, Kraft, \& Burdette, 2003) using the anatomic regions from the automatic anatomic labeling system (Tzourio-Mazoyer et al., 2002). We included three sets of regions: the amygdala, the dmPFC, and the vmPFC. Left and right amygdala comprised the amygdalar ROI. Left and right superior medial frontal cortex regions comprised the dmPFC ROI. Left and right medial orbital frontal and anterior cingulate cortex comprised the vmPFC ROI. For all reported effects, peak voxels of clusters surviving the statistical threshold are reported in Montreal Neurological Institute (MNI) space. Where graphed, neural activity for peak voxels shows the parameter estimates for the convolved regressors presented in arbitrary units.

\section{Results}

\section{Behavioral results}

The proportion of correct impressions, incorrect impressions, and new responses (misses) to the studied items as well as the proportions of correct rejections (CR) and positive and negative false alarms (FA) to unstudied items are shown in Table 1. To assess memory performance, we calculated impression memory by taking the proportion of correct impression responses out of the correctly recognized old faces, formula: correct impressions/(correct impressions + incorrect impressions). This analysis is an impression memory estimate we have used previously (Leshikar \& Gutchess, 2015; Leshikar et al., 2014). To assess whether the level of self-similarity 
Table 1 Proportion of studied items associated with correct impression, incorrect impression, and new responses (misses), as well as the proportions of new items endorsed as positive (FA-positive), negative (FA-Neg) or new (CR)

\begin{tabular}{|c|c|c|c|}
\hline & \multicolumn{3}{|l|}{ Impression Memory } \\
\hline & Correct impression & Incorrect impression & New (Miss) \\
\hline \multicolumn{4}{|c|}{ Studied items as a function of: } \\
\hline \multicolumn{4}{|l|}{ Self-similarity } \\
\hline High & $0.67(0.13)$ & $0.16(0.08)$ & $0.17(0.12)$ \\
\hline Medium & $0.63(0.09)$ & $0.18(0.07)$ & $0.19(0.10)$ \\
\hline Low & $0.60(0.10)$ & $0.20(0.09)$ & $0.20(0.09)$ \\
\hline \multirow[t]{2}{*}{ Unstudied items } & FA-Positive & FA-Negative & New (CR) \\
\hline & $0.24(0.14)$ & $0.12(0.08)$ & $0.64(0.22)$ \\
\hline
\end{tabular}

Note. Impression memory was calculated as the proportion of correct impression out of the proportion of correctly recognized old items (correct impression plus incorrect impression judgments).

(high, medium, low) affected memory, we performed a oneway ANOVA with polynomial contrasts to determine if there was a linear trend in impression memory performance. ANOVA results indicated a linear effect of self, $F(2,34)=$ $4.3, p=.05, \eta^{2}=.20$. Pairwise comparisons showed that impression memory was better for the high than for the low self-similarity trials, $t(17)=2.07, p=.05$. The medium self trials did not differ from either the high or low self trials, $t(17) \mathrm{s}$ $<1.24, p \mathrm{~s}>.23$.

\section{fMRI results}

We present two functional neuroimaging results: First, we report brain activity supporting impression formation of targets varying in similarity to the self (high, medium, low), regardless of subsequent memory. We were interested in regions showing increasing activity when thinking about targets more similar to the self, and the reverse. Second, we report regions supporting subsequent impression memory for others varying in similarity to the self. For each comparison, we performed additional analyses contrasting low-similarity trials (heretofore known as "dissimilar trials") with all other trials (high and medium similarity trials, heretofore known as "similar trials"), which is analogous to dissimilar versus similar others contrasts (and vice versa) employed in prior studies (Jenkins et al., 2008; Mitchell et al., 2006; Moore et al., 2014). ${ }^{4}$

\footnotetext{
${ }^{4}$ Specifically, we chose to bin high and medium similarity together because this was more equivalent to prior operational definitions of "similar others." For example, in Jenkins et al. (2008), the "similar" target they used was given an average rating by participants of 4.8 on a 7-point Likert scale $(1=$ most dissimilar [to self]; $7=$ most similar [to self]), which would be akin to a medium self-similarity trial on our scale. Because of this, we think our binning of the medium trials with the high self trials was a much better parallel to what has previously been used as "similar" target.
}

\section{Impression formation analysis}

Our first fMRI analysis examined activity during the impression formation task, regardless of memory performance. Starting first with regions showing increasing activity to similar others (high $>$ medium $>$ low), we found one cluster in the right dACC $($ MNI coordinate: $6,8,40 ; t$ value $=3.92$, BA 24, cluster size $=55$ ) showing increasing activity as self-similarity increased. Activity in this cingulate region is consistent with work showing involvement when thinking about others (Decety \& Sommerville, 2003; Northoff et al., 2006). For the reverse comparison (low $>$ medium $>$ high), we found activity in two predominantly visual processing regions in the right lingual gyrus (MNI coordinate: 24, $-76,16$; $t$ value $=3.68$, BA $18 / 19$, cluster size $=53$ ) and the left fusiform $(M N I$ coordinate: $-33,-52,-2 ; t$ value $=3.42$, BA 37 , cluster size $=19){ }^{5}$ Mean parameter estimates (betas) from the peak voxel of the right dACC and the left fusiform gyrus are shown in Fig. 2.

We performed an additional analysis to examine activity when the high and medium self-similarity trials were collapsed together and were contrasted against the low selfsimilarity trials, which is analogous to contrasts used in prior investigations. As expected, whole brain results indicated greater vmPFC activity when thinking about similar relative to dissimilar others (see Table 2). Because participants were forming both positive and negative impressions, we also examined regions showing valence effects (positive $>$ negative impression trials; negative $>$ positive). No regions emerged

\footnotetext{
${ }^{5}$ We found these primary effects held even when we inclusively masked these effects with both the high $>$ medium and the medium $>$ low contrasts. To perform this extra analysis, we used an inclusive mask thresholded at $p=0.1$, as was done recently (Liljeholm, Tricomi, O'Doherty, \& Balleine, 2011). By using this inclusive masking procedure (for both the high $>$ medium and medium $>$ low contrasts), we attained an effective inclusive mask of 0.01 (Friston, Holmes, Price, Büchel, \& Worsley, 1999; Price \& Friston, 1997).
} 


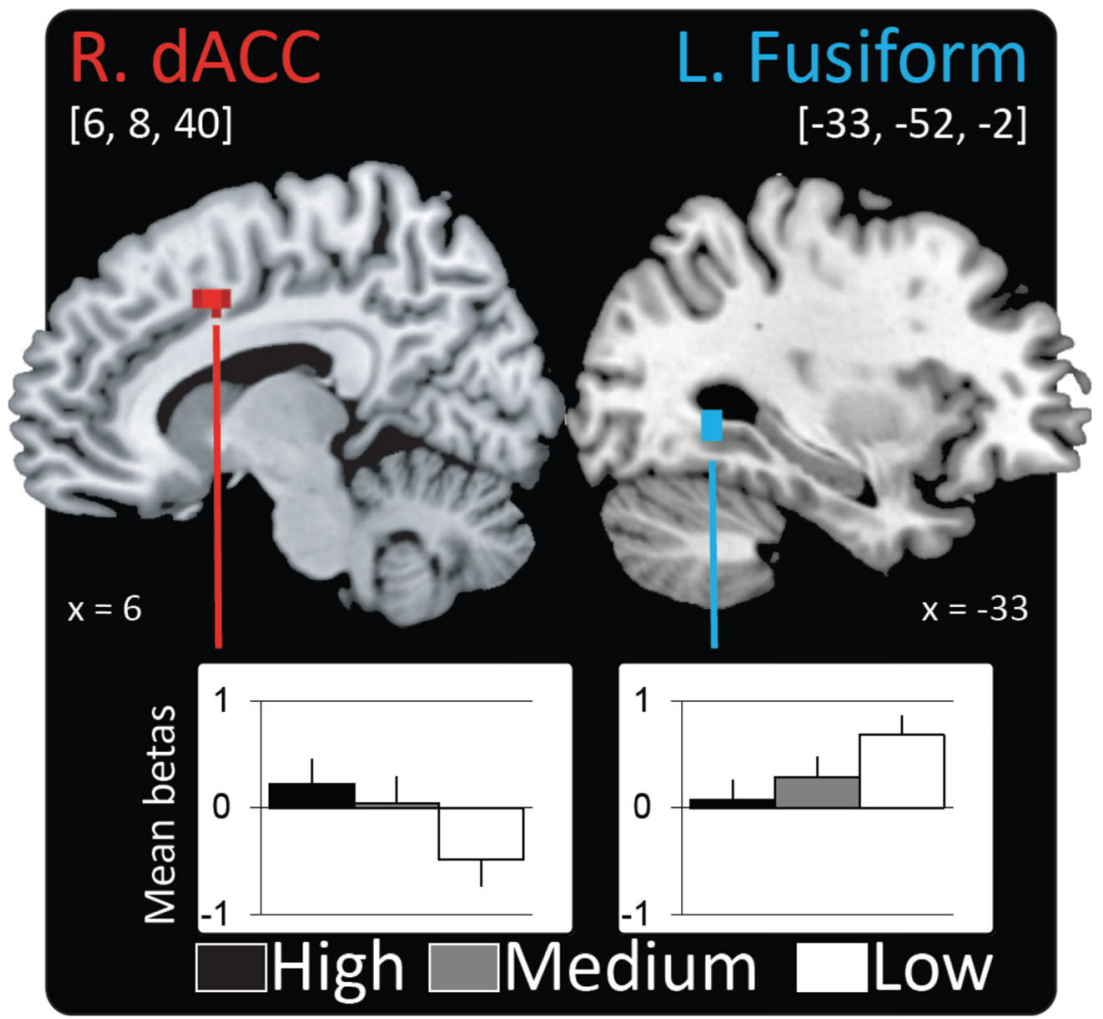

Fig. 2 Regions supporting impression formation of targets differing in similarity to the self. Activity in the right dACC (red) showed increasing activity to targets increasingly similar to the self (high $>$ medium $>$ low). By contrast, activity in the left fusiform (blue) showed increasing activity to targets decreasingly similar to the self (low $>$ medium $>$ high). Contrasts

from this comparison, suggesting that valence did not unduly influence neural activity as a function of self-similarity.

\section{Impression memory analysis}

Our second analysis examined regions supporting subsequent memory across self-similarity. To do this, we examined are rendered on a standard brain in Montreal Neurological Institute space. Bars represent mean parameter estimates (betas) for self-similarity conditions. Error bars depict the standard errors of the means. R. dACC $=$ right dorsal anterior cingulate cortex

regions showing better memory for high relative to medium relative to low similarity others, that is, (High_correct $>$ High_Incorrect $)>($ Medium_correct $>$ Medium incorrect $)>$ (Low correct $>$ Low incorrect). Results indicated one region in the precuneus survived this contrast (MNI coordinate: -12, 40,$7 ; t$ value $=3.69$, BA 29, cluster size $=33$ ). Because of our a priori interest for this contrast in the $\mathrm{dmPFC}, \mathrm{vmPFC}$ and the

Table 2 Regions showing greater activity while forming impressions for similar others (high and medium self-similarity) relative to dissimilar others (low self-similarity), regardless of memory performance

\begin{tabular}{|c|c|c|c|c|c|}
\hline Region & Hemisphere & MNI coordinates & $\mathrm{BA}$ & $t$ value & Cluster size \\
\hline Dorsal anterior cingulate cortex (dACC) & Right & 6840 & 24 & 4.13 & 98 \\
\hline Supplemental motor area & Left & -62352 & 8 & 3.45 & \\
\hline Caudate nucleus & Right & 9216 & NA & 4.03 & 28 \\
\hline Supplemental motor area & Left & $-6-155$ & 6 & 3.95 & 41 \\
\hline Inferior frontal gyrus & Left & -51201 & 45 & 3.48 & 27 \\
\hline Insula & Left & $-4217-2$ & 48 & 3.11 & \\
\hline Ventral medial prefrontal cortex & Right & 6531 & 10 & 3.42 & 24 \\
\hline Operculum & Left & -6057 & 48 & 3.35 & 32 \\
\hline Operculum & Left & $-63-113$ & 48 & 3.29 & \\
\hline
\end{tabular}

Note. The dACC region depicted in bold is the same region surviving the high $>$ medium $>$ low analysis shown in Fig. 2. Regions are listed for each cluster from highest to lowest $t$ value. Regions listed without a cluster size are subsumed by the larger cluster listed directly above. BA: Brodmann's area; MNI: Montreal Neurological Institute. 
amygdala, we performed a small-volume correction analysis to test for effects within these regions. No clusters survived the small-volume correction in dmPFC, vmPFC or the amygdala. The reverse contrast, that is, (Low_correct $>$ Low_incorrect) $>$ (Medium_correct $>$ Medium_incorrect) $>$ (High_correct $>$ High_incorrect) yielded hippocampus/parahippocamus activity (MNI coordinate: $27,-25,14 ;$ value $=3.69$, BA 29, cluster size $=33$ ) and two other regions, (left thalamus: MNI coordinate: $-18,-13,10 ; \mathrm{t}$-value $=4.07$, cluster size $=29$ ); (right central gyrus: $\mathrm{MNI}$ coordinate: $45,-4,34$; $\mathrm{t}$-value $=3.60$, BA 6 , cluster size $=24$ ).

We performed an additional set of analyses to examine memory for the impressions of similar (high/medium) relative to dissimilar (low) others, which is analogous to prior studies (Benoit et al., 2010; Jenkins et al., 2008; Mitchell et al., 2006; Moore et al., 2014). To do this, we first examined regions supporting memory for self-similar relative to dissimilar others, (high/medium impression correct > high/medium impression incorrect) $>$ (low impression incorrect $>$ low impression correct). There were no significant whole brain effects. We performed a smallvolume correction analysis to test for effects within these regions. We found that the right amygdala (MNI coordinate: $27,2,-27, t=4.30$, FWE-corrected $p=$ .005) supported impression memory for those similar to the self, consistent with prior work (Schiller et al., 2009). No clusters survived the small-volume correction in dmPFC or vmPFC. In a whole brain analysis, impression memory for those dissimilar to the self (low impression correct $>$ low impression incorrect) $>$ (high/medium impression incorrect $>$ high/medium impression correct) was supported by several regions including the hippocampus/parahippocampus (see Table 3). Mean parameter estimates (betas) from the peak voxel of the right amygdala and the right hippocampus/parahippocampus are shown in Fig. 3.

\section{Discussion}

This study examined regions responsive to thinking about others differing in degrees of self-similarity. More specifically, we examined regions involved while forming impressions of others who differed in self-similarity (high, medium, low) as well as regions supporting memory for these same target individuals. We report two primary findings. First, we found that a dorsal midline region, dACC, supported evaluations of others who differed in self-similarity. Specifically, dACC activity increased with increasing levels of self-similarity of targets. This suggests involvement of dACC in a process of comparing the self to others. Second, we found a double dissociation in regions supporting memory, with the amygdala supporting memory for similar targets and the hippocampus supporting memory for dissimilar targets. These data suggest that selfsimilarity influences the neural resources engaged when thinking about others and suggests a type of self-reference effect (Leshikar, Dulas, \& Duarte, 2015; Rogers, Kuiper, \& Kirker, 1977; Symons \& Johnson, 1997) in memory for others.

Our first goal was to identity neural regions supporting evaluations of others who differed in similarity to the self. While participants formed impressions, the dACC showed more activity to targets as similarity to the self increased. Importantly, this is the first work that shows that dorsal midline regions respond to different levels of self-similarity in target individuals (although, see Benoit et al., 2010). This complements prior work showing that dorsal midline activity supports social comparison between two social targets or between the self and a target (Hughes \& Beer, 2012; Lindner, Hundhammer, Ciaramidaro, Linden, \& Mussweiler, 2008). In particular, our data suggest that $\mathrm{ACC}$ is involved in an online process that codes for how similar social targets are to the self. Although speculative, it may be that this region is involved in "simulating" the behaviors of target individuals during impression formation. Specifically, because participants based

Table 3 Regions supporting impression memory for dissimilar others (low self-similarity) relative to similar others (high and medium self-similarity)

\begin{tabular}{|c|c|c|c|c|c|}
\hline Region & Hemisphere & MNI coordinates & BA & $t$ value & Cluster size \\
\hline Caudate nucleus & Left & $-18-1310$ & NA & 4.90 & 43 \\
\hline White matter/Caudate nucleus & Right & 9219 & NA & 4.56 & 36 \\
\hline Hippocampus/Parahippocampus & Right & $27-25-14$ & 20 & 4.48 & 39 \\
\hline Precentral gyrus & Right & $45-434$ & 6 & 3.64 & 33 \\
\hline Precentral gyrus & Right & $51-146$ & 6 & 3.55 & \\
\hline Lingual gyrus & Right & $3-6710$ & 17 & 3.64 & 63 \\
\hline Lingual gyrus & Right & $6-584$ & 18 & 3.18 & \\
\hline Precuneus & Left & $-12-674$ & 27 & 2.93 & \\
\hline Operculum & Right & $60-1013$ & 22 & 3.50 & 22 \\
\hline
\end{tabular}

Note. The hippocampal/parahippocampal region depicted in bold is shown in Fig. 3. Regions are listed for each cluster from highest to lowest $t$ value. Regions listed without a cluster size are subsumed by the larger cluster listed directly above. BA: Brodmann's area; MNI: Montreal Neurological Institute. 


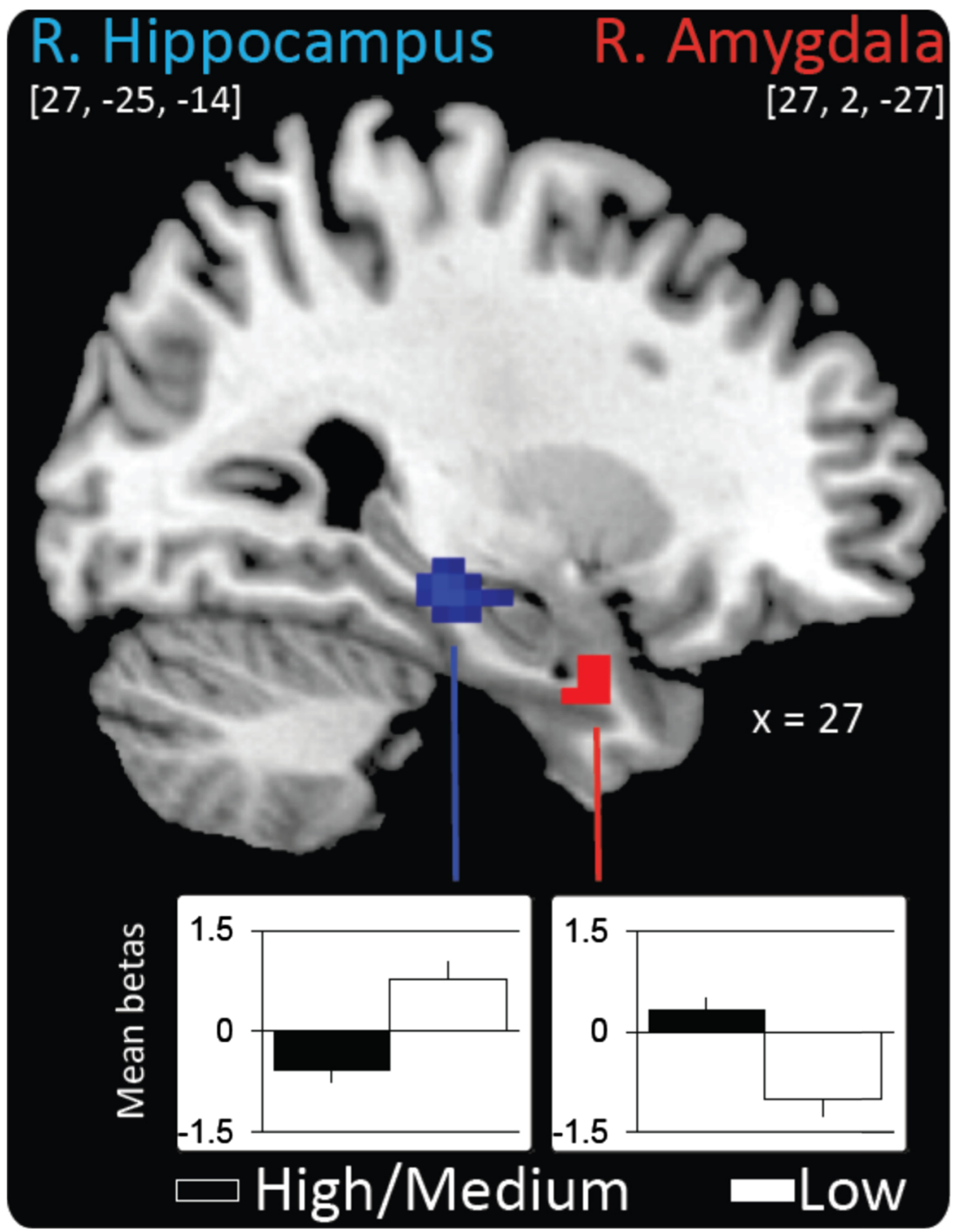

Fig. 3 Double dissociation in regions supporting memory for targets differing in self-similarity. Activity in the right hippocampus/ parahippocampus (blue) supported impression memory for targets dissimilar to the self. In contrast, activity in the right amygdala (red)

impression evaluations on behaviors, participants may have been simulating these behaviors. It may be, then, that activity of this region is most robust when encountering someone similar to self for whom simulations might be performed easily. Indeed, prior work suggests that dACC is essential in tasks that involve simulating the behaviors of targets (Blakemore \& Decety, 2001; Decety \& Sommerville, 2003). Although $\mathrm{dACC}$ has been associated with many other types of processes, a simulation account is particularly fitting given that participants were processing information about behaviors in this impression formation task. Because this is speculative, future work will be necessary to vet this possibility.

There is an alternative possibility of what the dACC activity might signify. There is some behavioral work suggesting that one aspect of the self-schema is "certainty" (e.g. I am very certain I am an outgoing person; Pelham, 1991). Interestingly, supported impression memory for targets similar to the self. Contrasts are rendered on a standard brain in Montreal Neurological Institute space. Bars represent mean parameter estimates (betas) for selfsimilarity conditions. Error bars depict the standard errors of the means

D'Argembeau et al. (2012) showed that this component of the self-schema is associated with dorsal (and not ventral) medial prefrontal activity. Given this prior work, it may be that the dACC activity we observed may have been most active when processing behaviors of others that were central components of the self (i.e., the high self-similarity trials). Thus, it may be that dACC was tracking self-other similarity for traits that are central to one's self schema rather than simulating those behaviors performed by others. In contrast to regions showing a positive relationship with self-similarity, we found that activity in fusiform gyrus and the lingual gyrus increased when thinking about others decreasing in self-similarity. One possible reason is that for the low similarity trials participants were paying more attention to faces when the behaviors were not as relevant to the self. Future work will be necessary to understand this. 
Prior work has largely implicated vmPFC activity when thinking about targets similar to oneself (Ebner et al., 2011; Mitchell et al., 2006; Moore et al., 2014). Consistent with this work, when we performed an analogous contrast (high/medium > low), we found that activity in the vmPFC supported thinking about similar others, which is a conceptual replication of that work. Data from the present study, taken together with prior work, provides additional evidence that vmPFC is involved when thinking about others most similar to the self but is less engaged by dissimilar others.

We also examined regions supporting memory for similar versus dissimilar others. Behaviorally we found that memory was better for self-similar relative to dissimilar others, suggesting a self-reference effect in person memory (Leshikar \& Gutchess, 2015). Our fMRI results revealed a dissociation in regions supporting memory dependent on self-similarity. For those similar to the self, activity in the amygdala supported subsequent impression memory, but for those dissimilar to the self, activity in the hippocampus was involved. This implicates the amygdala in long-term memory formation when processing social information that is especially relevant to oneself. Although most investigations of self-reference have been linked to activity in other regions (medial prefrontal cortex), there is some work that suggests that the amygdala does show heightened recruitment to self-relevant information (N'Diaye, Sander, \& Vuilleumier, 2009; Rameson et al., 2010; Yoshimura et al., 2009), as we observed in this study. Furthermore, the amygdala has more generally been shown to play a role in memory for social materials. For instance, Somerville and colleagues (2006), showed that face-behavior memory was strongly supported by the amygdala, but only when the behaviors were affectively rich. Furthermore, Cassidy and Gutchess (2012b) found that cortical volume of the amygdala was tied to memory performance for face-trait pairs in healthy older adults, whereas hippocampal volume was not. Although our data clearly suggests that the amygdala supports impression memory, it is still unclear exactly how this region might be doing so. One possibility is that encountering someone similar to the self is an affectively arousing experience triggering the involvement of amygdala. Such a mechanism is consistent with the role the amygdala plays for affective information (Adolphs, 2002; Buchanan \& Adolphs, 2002).

In contrast to the amygdala activity supporting memory for similar others, we found hippocampal activity supported memory most robustly for low self-similarity targets. One possible reason for this is that as self-similarity decreased, participants' attention may have been less directed to socially relevant aspects of the stimuli, leading to greater hippocampal involvement. Supporting this idea, in an experiment where participants processed social materials (face-behavior pairs) in either an impression formation task (infer what this person is like) or a sequencing task (remember the order the people appear in), Mitchell and colleagues (2004) found that memory was supported by the hippocampus in the sequencing task but not in the impression formation task. This evidence supports the idea that the hippocampus can support memory for social materials, but only under processing conditions that minimizes attention to the socioemotional nature of stimuli. Considering the amygdala and hippocampal findings together, these data suggests that as social information is more relevant to oneself, the hippocampus may play a diminished role in memory. Although this idea is at odds with the traditional conception that the hippocampus is a domain-general memory region (i.e., it binds information together regardless of the encoding process one engages in or the stimulus domain), it is compatible with neuropsychological and neuroimaging work showing that the hippocampus is not necessary to remember social material, although the amygdala is (Gilron \& Gutchess, 2012; Johnson, Kim, \& Risse, 1985; Todorov \& Olson, 2008). In sum, these data suggest an important role for the amygdala when remembering someone similar to the self.

It was surprising that dmPFC activity did not support memory in this investigation given prior work showing dmPFC support for impression memory (Gilron \& Gutchess, 2012; Mitchell et al., 2004) and memory for self-relevant material (Leshikar \& Duarte, 2012; Leshikar \& Duarte, 2014). One possible reason we did not see activity in this region is because we contrasted impression formation trials against one another. If dmPFC is associated with impression memory as suggested previously, we may have subtracted out impression memory related contributions insensitive to levels of self-similarity. That is, perhaps the dmPFC may be separately sensitive to self-similarity as well as memorability, but the combination of the two factors does not further modulate activity in the region. Future work will be necessary to test this prospect.

Although we found regions sensitive to self-similar others suggesting an online self-other comparison process, there are a few limitations to this study that are worth noting. First, because participants were aware of the memory test while forming impressions, these results may not generalize to incidental impression formation. We note, however, that because all of our trial types were studied under the same intentional encoding instructions, it is unlikely that encoding instruction would meaningfully interact with levels of self-similarity. Second, because of insufficient numbers of trials, we were not able to investigate how valence interacted with selfsimilarity or memory. This is important because prior work has shown that people exhibit strong self-enhancements biases (seeing positive traits associated with the self more so than negative; D'Argembeau \& Van der Linden, 2008; Leary, 2007; Leshikar \& Gutchess, 2015; Leshikar et al., 2014; Sedikides \& Green, 2000), and thus neural recruitment to trials associated with positive impressions may be neurally distinct from negative impressions. Future work will be necessary to examine how valence of the initial impression might 
interact with self-similarity. Third, it could be argued that the analysis we deployed to investigate self-similarity effects (high $>$ medium $>$ low) was akin to a high $>$ low similarity comparison. Although this is possible, additional analyses showed that our primary effects of interest held even when we inclusively masked these effects with both high $>$ medium and the medium $>$ low effects, arguing against this potential concern.

Overall, this study provides behavioral and neural evidence for the importance of self-similarity in considering how people think about others. Because people live in complex social structures, it is a given that we are often engaged in making evaluations of similar and dissimilar others, as well as those that fall in between these extremes. Our data suggest that selfsimilarity influences the memorability of targets implying a type of self-reference effect in person memory and that disparate cortical regions respond to thinking about others varying in self-similarity.

Acknowledgments We would like to thank Liat Zabludovsky and Pete Millar for help during data collection; Ryan Leach for helpful comments on an earlier version of this manuscript; as well as Amy Summerville, who gave thoughtful conceptual advice. E. D. Leshikar was supported by NIH Grant T32AG000204 while working on this project. This project was supported by NIA grant R21 AG032382 to A. H. Gutchess.

\section{References}

Adolphs, R. (2002). Neural systems for recognizing emotion. Current Opinion in Neurobiology, 12(2), 169-177.

Amodio, D. M., \& Frith, C. D. (2006). Meeting of minds: The medial frontal cortex and social cognition. Nature Reviews: Neuroscience, $7(4), 268-277$

Aron, A., Aron, E. N., \& Smollan, D. (1992). Inclusion of Other in the Self Scale and the structure of interpersonal closeness. Journal of Personality and Social Psychology, 63(4), 596.

Benoit, R. G., Gilbert, S. J., Volle, E., \& Burgess, P. W. (2010). When I think about me and simulate you: Medial rostral prefrontal cortex and self-referential processes. NeuroImage, 50(3), 1340-1349. doi: 10.1016/j.neuroimage.2009.12.091

Berscheid, E., Snyder, M., \& Omoto, A.M., 1989. Issues in studying close relationships: Conceptualizing and measuring closeness. In: C. Hendrick (Ed.) Close relationships. Review of personality and social psychology, Volume 10 (pp. 63-91). Thousand Oaks, CA, US: Sage Publications, Inc., p 270

Blakemore, S.-J., \& Decety, J. (2001). From the perception of action to the understanding of intention. Nature Reviews Neuroscience, 2(8), $561-567$.

Bogardus, E. S. (1933). A social distance scale. Sociology \& Social Research, 17, 265-271.

Bruch, M. A., Kaflowitz, N. G., \& Berger, P. (1988). Self-schema for assertiveness: Extending the validity of the self-schema construct. Journal of research in personality, 22(4), 424-444.

Buchanan, T. W., \& Adolphs, R. (2002). The role of the human amygdala in emotional modulation of long-term declarative memory. In S. C. Moore \& M. Oaksford (Eds.), Emotional cognition: From brain to behaviour (pp. 9-34). Philadelphia, PA: John Benjamins.

Cassidy, B. S., \& Gutchess, A. H. (2012a). Social relevance enhances memory for impressions in older adults. Memory, 20(4), 332-345.
Cassidy, B. S., \& Gutchess, A. H. (2012b). Structural variation within the amygdala and ventromedial prefrontal cortex predicts memory for impressions in older adults. Frontiers in Psychology, 3, 319.

Catrambone, R., Beike, D., \& Niedenthal, P. (1996). Is the self-concept a habitual referent in judgments of similarity? Psychological Science, $7(3), 158-163$.

Catrambone, R., \& Markus, H. (1987). The role of self-schemas in going beyond the information given. Social Cognition, 5(4), 349-368.

Cohen, N. J., Ryan, J., Hunt, C., Romine, L., Wszalek, T., \& Nash, C. (1999). Hippocampal system and declarative (relational) memory: Summarizing the data from functional neuroimaging studies. Hippocampus, 9(1), 83-98.

D’Argembeau, A., Jedidi, H., Balteau, E., Bahri, M., Phillips, C., \& Salmon, E. (2012). Valuing one's self: Medial prefrontal involvement in epistemic and emotive investments in self-views. Cerebral Cortex, 22(3), 659-667. doi:10.1093/cercor/bhr144

D'Argembeau, A., Ruby, P., Collette, F., Degueldre, C., Balteau, E., Luxen, A., ... Salmon, E. (2007). Distinct regions of the medial prefrontal cortex are associated with self-referential processing and perspective taking. Journal of Cognitive Neuroscience, 19(6), 935944.

D'Argembeau, A., \& Van der Linden, M. (2008). Remembering pride and shame: Self-enhancement and the phenomenology of autobiographical memory. Memory, 16(5), 538-547. doi:10.1080/ 09658210802010463

Decety, J., \& Sommerville, J. A. (2003). Shared representations between self and other: A social cognitive neuroscience view. Trends in Cognitive Sciences, 7(12), 527-533.

Denny, B. T., Kober, H., Wager, T. D., \& Ochsner, K. N. (2012). A metaanalysis of functional neuroimaging studies of self-and other judgments reveals a spatial gradient for mentalizing in medial prefrontal cortex. Journal of Cognitive Neuroscience, 24(8), 1742-1752.

Duarte, A., Henson, R. N., \& Graham, K. S. (2008). The effects of aging on the neural correlates of subjective and objective recollection. Cerebral Cortex, 18(9), 2169-2180. doi:10.1093/cercor/bhm243

Dulas, M. R., \& Duarte, A. (2011). The effects of aging on materialindependent and material-dependent neural correlates of contextual binding. NeuroImage, 57(3), 1192-1204.

Ebner, N. C., Gluth, S., Johnson, M. R., Raye, C. L., Mitchell, K. J., \& Johnson, M. K. (2011). Medial prefrontal cortex activity when thinking about others depends on their age. Neurocase, 17(3), 260-269.

Eichenbaum, H. (2000). A cortical-hippocampal system for declarative memory. Nature Reviews Neuroscience, 1(1), 41-50.

Fong, G. T., \& Markus, H. (1982). Self-schemas and judgments about others. Social Cognition, 1(3), 191-204.

Friston, K. J., Ashburner, J., Kiebel, S., Nichols, T. E., \& Penny, W. D. (Eds.). (2007). Statistical parametric mapping: The analysis of functional brain images. Waltham, MA: Elsevier.

Friston, K. J., Glaser, D. E., Henson, R. N., Kiebel, S., Phillips, C., \& Ashburner, J. (2002). Classical and Bayesian inference in neuroimaging: Applications. NeuroImage, 16(2), 484-512. doi:10.1006/ nimg.2002.1091

Friston, K. J., Holmes, A. P., Price, C., Büchel, C., \& Worsley, K. (1999). Multisubject fMRI studies and conjunction analyses. NeuroImage, 10(4), 385-396.

Gilron, R., \& Gutchess, A. H. (2012). Remembering first impressions: Effects of intentionality and diagnosticity on subsequent memory. Cognitive, Affective, \& Behavioral Neuroscience, 12(1), 85-98.

Henson, R. (2007). Efficient experimental design for fMRI. In K. J. Friston, J. Ashburner, S. Kiebel, T. E. Nichols, \& W. D. Penny (Eds.), Statistical parametric mapping: The analysis of functional brain images (pp. 193-210). Waltham, MA: Elsevier.

Hughes, B. L., \& Beer, J. S. (2012). Medial orbitofrontal cortex is associated with shifting decision thresholds in self-serving cognition. NeuroImage, 61(4), 889-898. 
Jenkins, A. C., Macrae, C. N., \& Mitchell, J. P. (2008). Repetition suppression of ventromedial prefrontal activity during judgments of self and others. Proceedings of the National Academy of Sciences, 105(11), 4507-4512.

Johnson, M. K., Kim, J. K., \& Risse, G. (1985). Do alcoholic Korsakoff's syndrome patients acquire affective reactions? Journal of Experimental Psychology: Learning, Memory, and Cognition, 11(1), 22.

Krienen, F. M., Tu, P. C., \& Buckner, R. L. (2010). Clan mentality: Evidence that the medial prefrontal cortex responds to close others. The Journal of Neuroscience, 30(41), 13906-13915.

Leary, M. R. (2007). Motivational and emotional aspects of the self. Annual Review of Psychology, 58, 317-344.

Leshikar, E. D., \& Duarte, A. (2012). Medial prefrontal cortex supports source memory accuracy for self-referenced items. Social Neuroscience, 7(2), 126-145.

Leshikar, E. D., \& Duarte, A. (2014). Medial prefrontal cortex supports source memory for self-referenced materials in young and older adults. Cognitive Affective \& Behavioral Neuroscience, 14, 236252. doi:10.3758/s13415-013-0198-y

Leshikar, E. D., Dulas, M. R., \& Duarte, A. (2015). Self-referencing enhances recollection in both young and older adults. Aging, Neuropsychology, and Cognition, 22(4), 388-412. doi:10.1080/ 13825585.2014.957150

Leshikar, E. D., \& Gutchess, A. H. (2015). Similarity to the self affects memory for impressions of others. Journal of Applied Research in Memory and Cognition, 22(4), 20-28.

Leshikar, E. D., Park, J. M., \& Gutchess, A. H. (2014). Similarity to the self affects memory for impressions of others in younger and older adults. The Journals of Gerontology Series B: Psychological Sciences and Social Sciences. doi:10.1093/ geronb/gbt132

Liljeholm, M., Tricomi, E., O’Doherty, J. P., \& Balleine, B. W. (2011). Neural correlates of instrumental contingency learning: Differential effects of action-reward conjunction and disjunction. The Journal of Neuroscience, 31(7), 2474-2480.

Lindner, M., Hundhammer, T., Ciaramidaro, A., Linden, D. E., \& Mussweiler, T. (2008). The neural substrates of person comparison-An fMRI study. NeuroImage, 40(2), 963-971.

Lombardo, M. V., Chakrabarti, B., Bullmore, E. T., Wheelwright, S. J., Sadek, S. A., Suckling, J., \& Baron-Cohen, S. (2010). Shared neural circuits for mentalizing about the self and others. Journal of Cognitive Neuroscience, 22(7), 1623-1635.

Maldjian, J. A., Laurienti, P. J., Kraft, R. A., \& Burdette, J. H. (2003). An automated method for neuroanatomic and cytoarchitectonic atlasbased interrogation of fMRI data sets. NeuroImage, 19(3), 12331239.

Markus, H., Smith, J., \& Moreland, R. L. (1985). Role of the self-concept in the perception of others. Journal of Personality and Social Psychology, 49(6), 1494.

Mende-Siedlecki, P., Baron, S. G., \& Todorov, A. (2013). Diagnostic value underlies asymmetric updating of impressions in the morality and ability domains. The Journal of Neuroscience, 33(50), 1940619415.

Miller, S. L., Celone, K., DePeau, K., Diamond, E., Dickerson, B. C., Rentz, D., ... Sperling, R. A. (2008). Age-related memory impairment associated with loss of parietal deactivation but preserved hippocampal activation. Proceedings of the National Academy of Sciences of the United States of America, 105(6), 2181-2186. doi: 10.1073/pnas.0706818105

Minear, M., \& Park, D. C. (2004). A lifespan database of adult facial stimuli. Behavior Research Methods, 36(4), 630-633.

Mitchell, J. P., Macrae, C. N., \& Banaji, M. R. (2004). Encoding-specific effects of social cognition on the neural correlates of subsequent memory. The Journal of Neuroscience, 24(21), 4912-4917.
Mitchell, J. P., Macrae, C. N., \& Banaji, M. R. (2006). Dissociable medial prefrontal contributions to judgments of similar and dissimilar others. Neuron, 50(4), 655-663.

Moore, W. E., Merchant, J. S., Kahn, L. E., \& Pfeifer, J. H. (2014). 'Like me?': Ventromedial prefrontal cortex is sensitive to both personal relevance and self-similarity during social comparisons. Social Cognitive and Affective Neuroscience, 9(4), 421-426.

Moran, J. M., Macrae, C. N., Heatherton, T. F., Wyland, C. L., \& Kelley, W. M. (2006). Neuroanatomical evidence for distinct cognitive and affective components of self. Journal of Cognitive Neuroscience, 18(9), 1586-1594. doi:10.1162/jocn.2006.18.9.1586

N'Diaye, K., Sander, D., \& Vuilleumier, P. (2009). Self-relevance processing in the human amygdala: Gaze direction, facial expression, and emotion intensity. Emotion, 9(6), 798.

Northoff, G., \& Bermpohl, F. (2004). Cortical midline structures and the self. Trends in Cognitive Sciences, 8(3), 102-107. doi:10.1016/j.tics. 2004.01.004

Northoff, G., Heinzel, A., de Greck, M., Bermpohl, F., Dobrowolny, H., \& Panksepp, J. (2006). Self-referential processing in our brain-A meta-analysis of imaging studies on the self. Neurolmage, 31(1), 440-457. doi:10.1016/j.neuroimage.2005.12.002

Northoff, G., Schneider, F., Rotte, M., Matthiae, C., Tempelmann, C., Wiebking, C., ... Heinze, H. J. (2009). Differential parametric modulation of self-relatedness and emotions in different brain regions. Human Brain Mapping, 30(2), 369-382.

Pelham, B. W. (1991). On confidence and consequence: The certainty and importance of self-knowledge. Journal of Personality and Social Psychology, 60(4), 518.

Phan, K. L., Taylor, S. F., Welsh, R. C., Ho, S.-H., Britton, J. C., \& Liberzon, I. (2004). Neural correlates of individual ratings of emotional salience: A trial-related fMRI study. NeuroImage, 21(2), 768-780.

Price, C. J., \& Friston, K. J. (1997). Cognitive conjunction: A new approach to brain activation experiments. NeuroImage, 5(4 Pt 1), 261270. doi:10.1006/nimg.1997.0269

Qin, P., \& Northoff, G. (2011). How is our self related to midline regions and the default-mode network? NeuroImage, 57(3), 1221-1233.

Rameson, L. T., Satpute, A. B., \& Lieberman, M. D. (2010). The neural correlates of implicit and explicit self-relevant processing. NeuroImage, 50(2), 701-708.

Rogers, T. B., Kuiper, N. A., \& Kirker, W. S. (1977). Self-reference and the encoding of personal information. Journal of Personality and Social Psychology, 35(9), 677-688.

Schiller, D., Freeman, J. B., Mitchell, J. P., Uleman, J. S., \& Phelps, E. A. (2009). A neural mechanism of first impressions. Nature Neuroscience, 12(4), 508-514.

Schneider, F., Bermpohl, F., Heinzel, A., Rotte, M., Walter, M., Tempelmann, C., ... Northoff, G. (2008). The resting brain and our self: Self-relatedness modulates resting state neural activity in cortical midline structures. Neuroscience, 157(1), 120-131.

Sedikides, C., \& Green, J. D. (2000). On the self-protective nature of inconsistency-negativity management: Using the person memory paradigm to examine self-referent memory. Journal of Personality and Social Psychology, 79(6), 906.

Slotnick, S. D., Moo, L. R., Segal, J. B., \& Hart, J., Jr. (2003). Distinct prefrontal cortex activity associated with item memory and source memory for visual shapes. Brain Research: Cognitive Brain Research, 17(1), 75-82.

Somerville, L. H., Wig, G. S., Whalen, P. J., \& Kelley, W. M. (2006). Dissociable medial temporal lobe contributions to social memory. Journal of Cognitive Neuroscience, 18(8), 1253-1265.

Symons, C. S., \& Johnson, B. T. (1997). The self-reference effect in memory: A meta-analysis. Psychological Bulletin, 121(3), 371-394.

Todorov, A., \& Olson, I. R. (2008). Robust learning of affective trait associations with faces when the hippocampus is damaged, but not when the amygdala and temporal pole are damaged. Social Cognitive and Affective Neuroscience, 3(3), 195-203. 
Todorov, A., \& Uleman, J. S. (2003). The efficiency of binding spontaneous trait inferences to actors' faces. Journal of Experimental Social Psychology, 39(6), 549-562.

Triandis, H. C., \& Triandis, L. M. (1960). Race, social class, religion, and nationality as determinants of social distance. The Journal of Abnormal and Social Psychology, 61(1), 110.

Tzourio-Mazoyer, N., Landeau, B., Papathanassiou, D., Crivello, F., Etard, O., Delcroix, N., ... Joliot, M. (2002). Automated anatomical labeling of activations in SPM using a macroscopic anatomical parcellation of the MNI MRI single-subject brain. NeuroImage, 15(1), 273-289. doi:10.1006/nimg.2001.0978

Wagner, D. D., Haxby, J. V., \& Heatherton, T. F. (2012). The representation of self and person knowledge in the medial prefrontal cortex. Wiley Interdisciplinary Reviews: Cognitive Science, 3(4), 451-470.

Yoshimura, S., Ueda, K., Suzuki, S., Onoda, K., Okamoto, Y., \& Yamawaki, S. (2009). Self-referential processing of negative stimuli within the ventral anterior cingulate gyrus and right amygdala. Brain and Cognition, 69(1), 218-225. doi:10.1016/j.bandc.2008.07.010 